\title{
FERRAMENTAS QUE AUXILIAM NO DESENVOLVIMENTO DE GESTÃO EM MICRO PEQUENAS EMPRESAS
}

\author{
TOOLS ASSISTING MANAGEMENT DEVELOPMENT IN MICRO SMALL \\ ENTERPRISES
}

Alisson Felippe Da Cruz1, Lucas Cassiano Firmo De Oliveira, Marcos Paulo Da Silva, Edilene Mayumi Murashita Takenaka

Universidadedo Oeste Paulista - UNOESTE, curso de Tecnologia em Gestão Comercial. e-mail : Alisson_Pp81@Live.com1

RESUMO - A Vivencia no mercado dispõe múltiplas empresas disponíveis para os consumidores, acirrando o mercado competitivo, assim o consumidor passa a buscar não apenas consumir e sim uma relação de consumo, onde as empresas tendem a se desenvolver internamente para uma constante evolução. A tomada de decisão interna de uma organização, reflete ao seu cenário externo sendo assim, o principal desafio é a melhoria continua, onde a existência desse estudo tem como objetivo discutir as ferramentas que auxiliam o desenvolvimento em micro e pequenas empresas e identificar ferramenta para o auxílio e gestão do processo continuo, podendo ser utilizada pelas microempresas para o seu crescimento interno. Onde optou-se pela abordagem metodológica qualitativa, sendo utilizado como instrumento de coleta e análise de informações, a pesquisa bibliográfica, onde foi possível concluir que dentre diversas ferramentas, a utilização do ciclo demming conhecido como ciclo PDCA são eficazes para o auxílio e controle de um processo de controle e melhoria continua.

Palavras-chave: controle; processo administrativo; Ciclo PDCA; melhoria continua.

ABSTRACT - The experience in the market has multiple companies available to consumers, stimulating the competitive market, so the consumer starts to seek not only to consume but a relationship of consumption where companies tend to develop internally for a constant evolution. The internal decision-making of an organization reflects its internal scenario and thus the main challenge is continuous improvement, where the existence of this study has the objective to discuss the tools that help the development in micro and small companies and identify tool for the aid and management of the continuous process and can be used by micro-enterprises for their internal growth. Where the qualitative methodological approach was used as a tool for collecting and analyzing information, it was possible to conclude that among several tools the

Recebido em: 09/08/2017 Revisado em: 22/09/2017 Aprovado em: 03/10/2017 use of the Deming cycle known as the PDCA cycle is effective for the aid and control of a process of improvement.

Keywords: control; administrative process; PDCA; continuous improvement. 


\section{INTRODUÇÃO}

As empresas familiares apesar de pouco estudadas, tende a ter grande relevância no cenário e os aspectos que envolvem seu modelo de gestão e uma notável participação na economia. Ela é constituída por integrantes da mesma família.

Empresas familiares tem suas próprias características que garantem uma vantagem competitiva, mas essas mesma características podem influenciam conflitos, e os conflitos atingem a relação familiar e a empresa, como uma situação em que necessita tomar uma decisão que afeta a família ou a empresa, podendo ter a interferência de ambas as partes.

[...]os laços de família constituem um fator, entre outros, que determina a sucessão nos cargos administrativos; esposas ou filhos dos atuais ou antigos dirigentes máximos encontra-se no conselho administrativo; as ações práticas de um membro da família, refletem-se na reputação da empresa, independentemente de sua ligação na administração; a posição do parente na firma, influi em sua situação na família; cada membro da família precisa chegar a um acordo, quanto as suas relações com a empresa ao determinar sua própria carreira a seguir(DONNELEY,1976, p. 4)

Segundo pesquisas realizadas pelo SEBRAE (2011) entre o ano 2009 a 2010, em torno de $58 \%$ das pequenas e micro empresas morrem nos seus primeiros cinco anos após sua abertura, e uma das principais causas é devido a má gestão que conduz os negócios. As Micro empresas precisam estar preparadas para desenvolver novas estratégias para assim conseguir aumentar sua capacidade de tomada de decisões importante para sua sobrevivência.

Para garantir a sobrevivência com eficiência, as empresas familiares precisam ser administradas com 0 maior profissionalismo possível, incluindo todos processos da empresa, que podem afligir as empresas familiares.

Os principais desafios consistem na sobrevivência, capitalização, gestão profissional e sucessão, além desse, outros constantes desafios a serem superados também são expostos e analisados no estudo.

O presente trabalho apresenta aplicações de ferramentas profissionais visando para organização familiar, podendo ser aplicada em diversas áreas administrativas. Sendo a problemática em que este artigo apresenta é responder a questão de : quais são as ferramentas que possam ser utilizadas para auxiliar a gestão de processos em micro e pequenas empresas familiares.

E por tanto o objetivo é identificar as ferramentas de melhoria continua e analisar a execução do ciclo PDCA. 


\section{METODOLOGIA}

A metodologia utilizada para este artigo foi através da abordagem qualitativa, que de acordo com DENZIN, N. K. e LINCOLN $(2006$, p.17) [...] é caracterizada por relação de aspectos não somente mensuráveis, mas também definidos descritivamente e usam estratégias diversas de investigação.

Justificando a escolha para este artigo que á o objetivo da compreensão do assunto e não mensurações variáveis.

A abordagem adotada para coleta de dados deste trabalho foi o estudo bibliográfico, que foi fundamentada e coletada informações realizadas junto a matérias prontas, como livros, jornais, revistas, internet. Como instrumento de pesquisa neste trabalho. Tendo em vista que, segundo Gomez (1994), é pela análise de conteúdo que se prossegue na descoberta para interpretação dos conteúdos, além das aparências do que está comunicado. Sendo assim permitiu a análise de dados aprofundando as informações.

A metodologia é uma disciplina que consiste em estudar e avaliar os vários métodos possíveis. É a melhor maneira para abordar determinados problemas em seu estado atua de conhecimento. É o estudo crítico do método (COSTA, 2001).

\section{RESULTADOS}

Nesta seção serão apresentados todos os resultados encontrados com esta pesquisa, realizada a partir de materiais já publicadas.

As primeiras atividades desenvolvidas com esta metodologia do ciclo PDCA ocorreu na década de 20, por Walter Shewhart, mas quem realmente o disseminou foi Edward Deming, no qual o ciclo também ficou conhecido por Ciclo de Deming. O ciclo PDCA é um método gerencial de tomadas de decisões para auxiliar no alcance das metas para sobrevivência de uma organização.

Como o próprio nome "ciclo" representa um processo sem fim, fazendo repetidamente um questionamento dos trabalhos detalhados de um determinado processo, o método representa um processo sem fim, gerando algo repetidamente com um trabalho em detalhes de um processo. Quando se conclui, ele inicia novamente, sendo assim, o ciclo PDCA é definido como a sequência de atividades analisadas para melhorar as práticas da organização empregada, em processos de fabricações ou outros processos de negócios da organização. Antigamente esse conceito era aplicado exclusivamente nos processos de fabricação, porém, hoje aplicado em empresas que passam a adotar o ciclo para diversos processos de organização, realizando também adaptações a cada perfil, 
fortalecendo o conceito de melhoria continua em todos níveis da empresa.

De acordo com Deming e Walton (1992 apud ANACLETO, 2004), as etapas do ciclo PDCA, são compostos por quatro etapas:

- Planejar

- $\quad$ Executar

- Checar

- $\quad$ Agir

As descrições das atividades a serem desenvolvidas em cada etapa da seguinte forma:

- Planejar: estabelece o plano, de acordo com as diretrizes de cada empresa, identificar o problema e estabelecer os objetivos e metas.

- Executar: colocar o plano em pratica, estabelecer métodos a serem utilizados e objetivos, coletar dados para verificação do processo.

- Checar: verificar o trabalho que está sendo executado, conforme o planejamento e os valores que devem ser mantidos sobre os padrões estabelecidos.

- $\quad$ Agir: ações para corrigir trabalhos ou tarefas que possam ter se desvirtuado do padrão, verificar as causas e tomar as devidas ações para não se repeti-las.

A figura apresenta uma representação em círculo do ciclo PDCA onde demonstram as etapas e passos de cada etapa estão representados.

Após o ciclo PDCA implantado, e as melhorias implementadas, pode-se utilizar para manter a qualidade.

Figura 1. Ciclo Demming PDCA
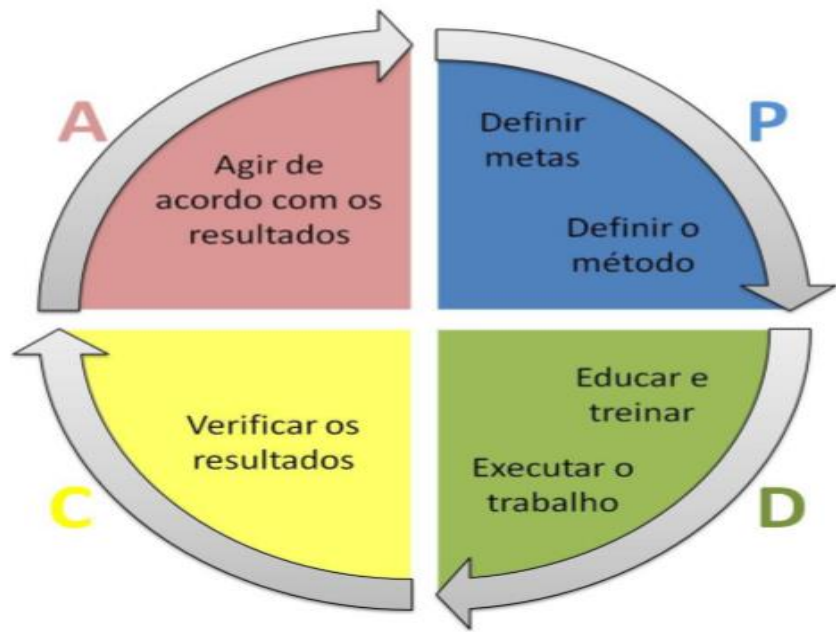

Fonte: Campos (2004). 
Figura 2. Detalhamento por setorização de processo aplicado.

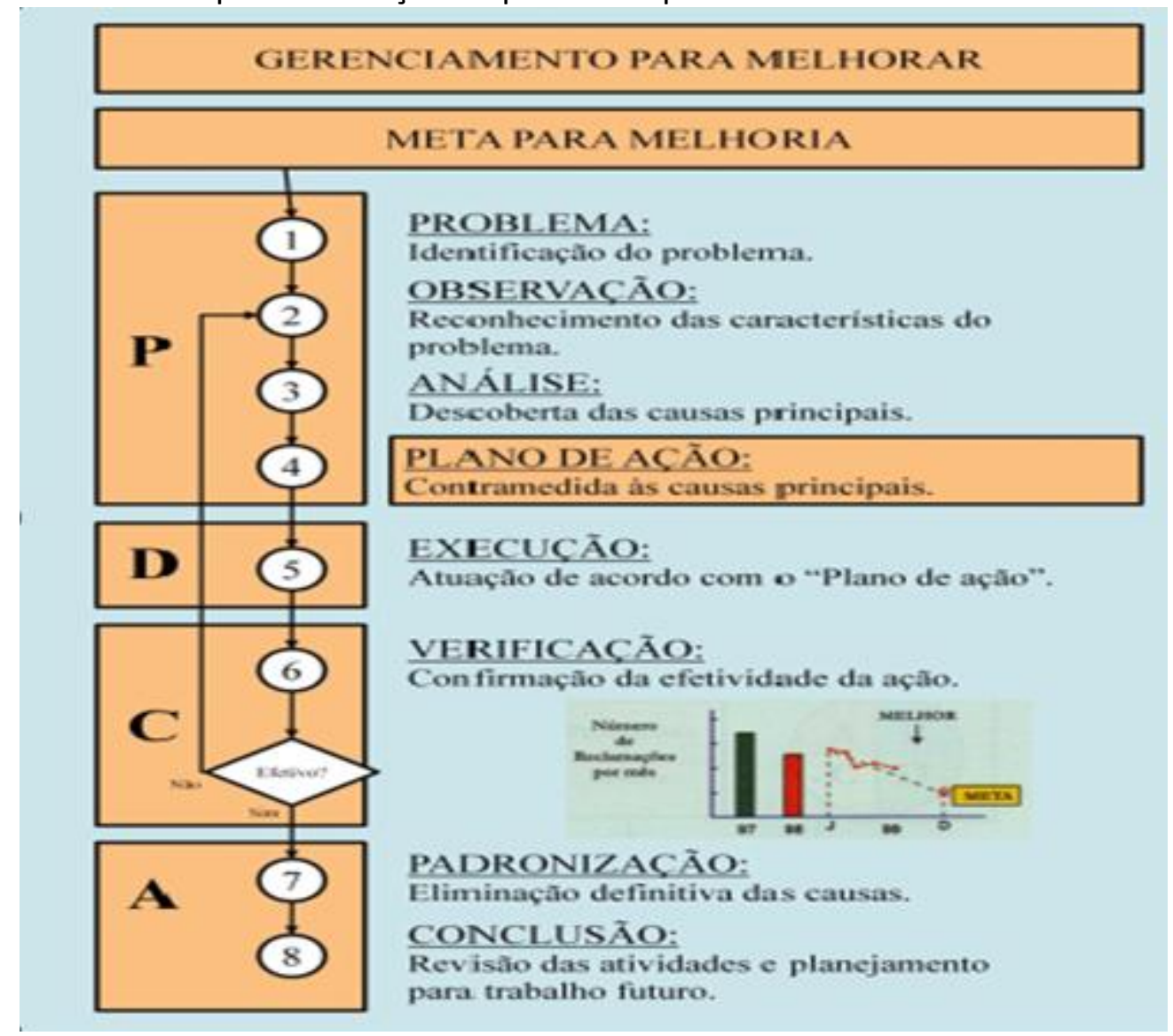

Fonte: Campos (2004)

Para o gerenciamento do ciclo no

primeiro ponto é importante identificar o principal problema a ser resolvido e observar todas suas características para que possa saber sua principal causa;
Sendo assim, o gestor coloca em execução onde aplicada o plano de ação e mantem em constante verificação de resultados para que possa obter dados positivos ou negativos. 
Figura 3. Aplicação com objetivo de melhoria continua

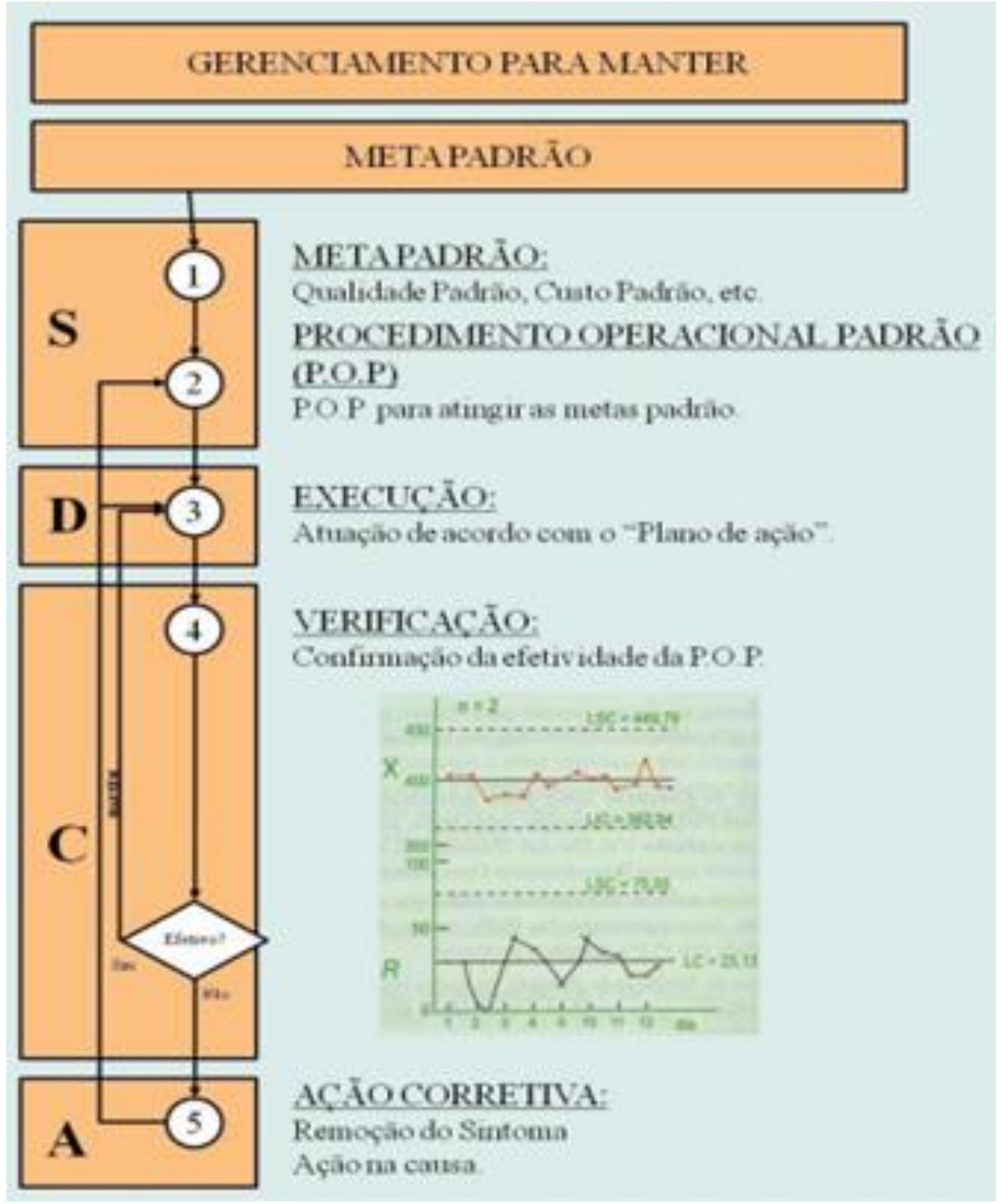

Fonte: Campos (2004).

A figura 3 representa de forma clara o SDCA que após executar o PDCA e encontrar o resultado esperado é preciso padronizar para que possa obter a melhoria continua

- $\quad$ S( standard) : É a padronização do processo para que todos executem a tarefa da mesma forma, criando procedimentos, instruções de trabalho .

- $\quad D(d o)$ : é a execução dos treinamentos, orientações de todos os envolvidos para a nova forma de fazer o trabalho.

- C ( check) : O Check relata o mesmo princípio do PDCA, verificar o cumprimento das tarefas conforme o padrão

- $\quad A(A c t)$ : Visa realizar ajustes necessários quando os colaboradores não conseguirem executar a nova forma do processo. 
Enfim, essa ferramenta acaba facilitando a estratégia para atribuir a melhoria em ambos setores, auxiliando um cronograma onde visa a facilidade de visualização de falhas no processo, atribuindo indicadores para solução.

As Microempresas que adotam o ciclo PDCA, atribuem melhorias no processo onde emprega, fazendo com que o processo seja revisado passando por uma análise e identificação, sendo realizado um reajuste no processo para solucionar a falha e atribuindo para um processo de melhoria continua onde a execução e a verificação seja constante. Com isso o alcance de melhoria dentro da organização empregada terá redução de custos, viabilidade e controle devido aos dados obtidos na utilização do ciclo PDCA.

\section{CONCLUSÃO}

O presente trabalho, demonstra por meio de pesquisa bibliográfica a utilização da ferramenta PDCA no relacionamento de controle e melhoria continua nos processos para microempresas, afim de utiliza-la em ambos processos para 0 auxílio 0 crescimento e sucesso na organização.

Durante o estudo, foi possível analisar que muitas organizações familiares acabam gerindo suas empresa de forma inabitado para o crescimento devido a falta de informação e qualificação dos gestores de sua empresa, não conhecendo as ferramentas básicas de auxílio a gestão de controle. Observa-se que entre ambas ferramentas o ciclo PDCA auxiliam em pleno processos aplicados internamente e externamente, buscando o controle de um processo falho e o crescimento organizacional.

Conclui-se que as pequenas e microempresas, quando possuem a aplicação e conhecimento da ferramenta na organização, possuem inúmeras oportunidades de desenvolvimento organizacional contribuindo ao crescimento e o controle do processo onde empregado

\section{REFERÊNCIAS}

ANACLETO, A. Método e modelo de avaliação para melhoria de processos de software em micro e pequenas empresas. Siddertação de Mestrado. UFSC, 2004. Disponível em: $<$ http://www.gqs.ufsc.br/wpcontent/uploads/2011/11/Dissertacao Alessandr aAnacleto.pdf> Acesso em: 11 jun. 2017.

CAMPOS, V. F. Controle da qualidade total. 3. ed. Rio de Janeiro: Bloch, 1992.

COSTA, Marco Antônio F. Metodologia da pesquisa conceitos e técnicas. Rio de Janeiro: Interciência. 2001.

DENZIN, N. K. e LINCOLN, Y. S. Introdução: a disciplina e a prática da pesquisa qualitativa. In: DENZIN, N. K. e LINCOLN, Y. S. (Orgs.). O planejamento da pesquisa qualitativa: teorias e abordagens. 2. ed. Porto Alegre: Artmed, 2006. p. 15-41.

DONNELEY, R. G. A empresa familiar. Biblioteca Harvard de Administração de Empresas. São Paulo: Abril-Tec, 1976. 
GOMES, R. A análise de dados em pesquisa qualitativa. In: MINAYO, M. C. de S. Pesquisa social: teoria, método e criatividade. Petrópolis, RJ: Vozes, 1994, p. 67-80.

SEBRAE Serviço Brasileiro de Apoio às Micro e Pequenas Empresas. Taxa de Sobrevivência das Empresas no Brasil. Coleção Estudos e Pesquisas, out.2011. Disponível em < https://m.sebrae.com.br/Sebrae/Portal\%20S ebrae/Anexos/Sobrevivencia das empresas no Brasil 2011.pdf>. Acesso em: 21 nov.2016. 